\title{
Platelet-rich Plasma
}

National Cancer Institute

\section{Source}

National Cancer Institute. Platelet-rich Plasma. NCI Thesaurus. Code C106557.

A plasma sample that is prepared from whole blood, which has undergone a number of centrifug ation steps to concentrate the amount of platelets. 